\title{
Ubiquitous Intelligence and computing for enabling a smarter world
}

\author{
Diego López-de-Ipiña ${ }^{1} \cdot$ Liming Chen ${ }^{2} \cdot$ Nathalie Mitton $^{3} \cdot$ Gang Pan $^{4}$
}

Published online: 21 February 2017

(C) Springer-Verlag London 2017

This special issue of the Personal and Ubiquitous Computing (PUC) journal is devoted to papers selected from the 13th IEEE International Conference on Ubiquitous Intelligence and Computing (UIC 2016), held in Toulouse, France, on 18-21 July 2016.

Ubiquitous sensors, devices, networks and information are paving the way towards a Smart World in which computational intelligence is distributed throughout the physical environment to provide reliable and relevant services to people. This Ubiquitous Intelligence will change the computing landscape, enabling new breeds of applications and systems to be developed, and the realm of computing possibilities will be significantly extended. By enhancing everyday objects with intelligence, many tasks and processes can be simple, and the physical spaces where people interact like the workplaces and homes could become more efficient, safer and more enjoyable.

Diego López-de-Ipiña

dipina@deusto.es

Liming Chen

liming.chen@dmu.ac.uk

Nathalie Mitton

nathalie.mitton@inria.fr

Gang Pan

gpan@zju.edu.cn

1 Deusto Institute of Technology, DeustoTech, University of Deusto, Avda. de las Universidades 24, Bilbao 48007, Spain

2 School of Computer Science and Informatics, De Montfort University, The Gateway, Leicester LE1 9BH, UK

3 Inria Lille-Nord Europe, 40 Avenue Halley, Lille, Villeneuve D’Ascq 59650, France

4 Department of Computer Science, Zhejiang University, Hangzhou 310027, P.R. China
Ubiquitous Computing, or Pervasive Computing, uses these many "smart things or u-things" to create smart environments, services and applications. Ubiquitous computing and digital applications are also the new engines of the global economy.

Ubiquitous Intelligence is an emerging research field covering many disciplines. A series of grand challenges exist to move from the current level of computing services to the Smart World of adaptive and intelligent services. With this in mind, this special issue of the Personal and Ubiquitous Computing journal has aimed to disseminate the latest research results on (1) the underpinning technologies of Smart World in the areas of Smart Objects and Interactions, Smart Systems and Services and Personalisation and Social Aspects which enable and support usercentric, context-aware, reactive, adaptive and personalised services for meeting users' everyday needs and demands; (2) the real deployments and experimentation of Smart World applications which explore and showcase the potential of combining Smart Objects (e.g. RFID, NFC, iBeacons), Smart Systems (e.g. Big Data, Context-aware systems) and Personalisation and Social Aspects (e.g. persuasive interfaces, social computing or behaviour change modelling) towards Smart Environments and Applications (e.g. Smart Healthcare, Ambient-assisted Working Environments or Urban Computing and Smart Cities).

Indeed, this special issue has particularly intended to produce evidence and the best RTD practice to support the following equation: Smarter World $=$ Smart Objects $+S$ mart Systems + Personalisation and Social Aspects. Concretely, the revised versions of the selected papers included in this special issue cover key areas to enable the Smarter World vision: (a) activity recognition as key element to produce smarter environments responsive to users' actual 
needs; (b) the increasing role of Big Data to enable a Smarter Word scenarios; (c) Semantic Web as an enabler of Smarter World solution; (d) positioning as a key attribute of context extensively used to offer smarter services to people in indoor environments; and (e) the energy domain as a key catalyser of Smarter World solutions sensitive to the planet's needs.

Activity recognition is capital to be able to understand what users are carrying out within a Smart Environment and enable the environment to respond or anticipate to users' needs appropriately. The article "Semantic Segmentation of Real-time Sensor Data Stream for Complex Activity Recognition" by Darpan Triboan et al. [1] tackles the key role played by data segmentation in human activity recognition (HAR) within the Ambient-Assisted Living (AAL) domain. It is particularly important for complex activity recognition when the events occur in short bursts with attributes of multiple sub-tasks. Although past efforts were made in segmenting the real-time sensor data stream such as static/dynamic window sizing approaches, little has been explored to use the description of the activity of daily living (ADL) to support generic/user-specific preferences at segmentation stage. Therefore, this paper proposes semantic-based segmentation approach which uses ontology to perform terminology-box (T-Box) and assertion-box (A-Box) reasoning, along with logical rules to infer whether the incoming sensor event is related to a given sequences of the activity. A use-case scenario is used to illustrate how the proposed approach conducts semantic segmentation of real-time sensor data stream to recognise elderly persons' complex activities.

Also within the activity recognition domain, the article "A Novel Orientation and Location-Independent Activity Recognition Method" by Dianxi Shi et al. [2] proposes a solution to deal with the challenge posed by the orientation and location of a mobile phone when used to perform activity recognition. The core contribution of this work is a dynamic coordinate transformation approach to eliminate the effects of orientation and location changes in a mobile device when using its accelerometer data as source to undertake activity recognition. A real-time system implemented in Android is illustrated to demonstrate good accuracy results obtained by the device location and position agnostic activity recognition method proposed.

Infrastructure-wise Big Data is a key enabler to enable smart solutions which so far have not been possible to meet high computing demands. Accordingly, in this special issue, the article "Data Fusion in Automotive Applications - Efficient Big Data Stream Computing Approach" by Amir Haroun et al. [3] presents an interesting use case where connected vehicles streaming huge amounts of data at very high frequencies are used for by diverse entities, namely automobile manufacturer, vehicles owners and so on, for diverse services ranging from road safety services to after-market services (e.g. predictive and preventive maintenance). The main contribution of this work is to analyse how to enhance performance of data fusion to support several millions of connected vehicles through a spatial indexation approach. Interestingly, the effectiveness of the proposal is showcased by conducting real experiments over the PSA-Group Big Data streaming platform.

In the same topic, the article "Designing Parallel Data Processing for Enabling Large-Scale Sensor Applications" by Milan Kabác et al. [4] proposes a design-driven approach to developing orchestrating applications for masses of sensors that integrates parallel processing of large amounts of data. Specifically, an application design exposes declarations that are used to generate a programming framework based on the MapReduce programming model. Their main contribution, namely a design language, is used to abstract over implementation details, while exposing architectural properties used to generate highperformance code for processing large data sets. The prototype is implemented over Apache Hadoop and it is tested over a case study. Authors report significant speedups by parallelizing computations over twelve nodes.

As another key enabler, Semantic Web customised to the performance demands of IoT environments is also tackled. The article "IoT-Lite: A Lightweight Semantic Model for the Internet of Things and its Use with Dynamic Semantics" by Maria Bermudez-Edo et al. [5] faces a twofold problem, firstly, most of the IoT-related semantic descriptions are not as widely adopted as expected and, secondly, semantic techniques usually increase the complexity and processing time and therefore they are unsuitable for dynamic and responsive environments such as the IoT. To address these concerns, authors propose IoT-Lite, an instantiation of the semantic sensor network (SSN) ontology to describe key IoT concepts allowing interoperability and discovery of sensory data in heterogeneous IoT platforms by a lightweight semantics. The scalability of IoT-Lite is demonstrated by providing some experimental analysis and assess IoT-Lite against another solution in terms of round trip time (RTT) performance for query-response times. Besides, they link IoT-Lite with Stream Annotation Ontology (SAO), to allow queries over stream data annotations.

The core issue of location as an essential contextual attribute is also explored in this special issue. The article "GraphLoc: A Graph-based Method for Indoor Subarea Localization with Zero-configuration" by Yuanyi Chen et al. [6] proposes a zero configuration method for localisation in indoor environments which avoids usual site survey and pre-installing additional infrastructure steps. For that purpose, authors utilise two un-exploited characteristics of Wi-Fi radio signal strength to generate logical 
floor graph and then formulate the problem of constructing fingerprint map as a graph isomorphism problem between logical floor graph and physical floor graph. Besides, a Bayesian-based approach is utilised to estimate the unknown subarea in the online localisation phase. The proposed method has been implemented in a real-world shopping mall, and extensive experimental results show that the proposed method can achieve competitive performance comparing with existing methods.

Besides, the article "Overcrowding Detection in Indoor Events using Scalable Technologies" by Unai LopezNovoa et al. [7] deals with how presence and location estimation can be used to detect overcrowding in indoors environments, e.g. conference and business events. To that end, leveraging on the availability of already connectable devices among attendees, a method to allow nonintrusive positioning during the event, without the need of specific tracking devices, is provided. As a result, an algorithm for overcrowding detection based on passive $\mathrm{Wi}$ Fi requests capture and a platform for event monitoring that integrates this algorithm, are contributed in this work. The resulting solution offers access control management, attendees monitoring and the analysis and visualisation of the captured information, using a scalable software architecture. The accuracy and scalability of the algorithm are tested over an Apache Spark-based environment.

Finally, the special issue deals with one of the key application domains of Smarter World solutions, i.e. energy management within public buildings. The article "Comparison of Detailed Occupancy Profile Generative Methods to Published Standard Diversity Profiles" by Dimosthenis Ioannidis et al. [8] tackles the challenge of how to perform more efficient energy management within public buildings. The aim of this work is to identify disparities between different occupancy estimation techniques; standardised occupancy profiles found in literature, business processes-based profiles through interviews and accurate profiles from real on-field measurements. The occupancy diversity profiles of secondary spaces in a healthcare facility building are analysed through descriptive statistics and $t$ test methods over different time horizons. The results indicate that the utilisation of real occupancy data, along with elaboration of the business processes that take place in building spaces, has the potential to support more precise profiles in Building Performance Simulation (BPS) software tools.

Also, the article "On-demand Energy Monitoring and Response Architecture in a Ubiquitous World" by Oihane Kamara-Esteban et al. [9] tackles the challenge of load balance through demand-response strategies as one of the most effective and immediate actions aimed at achieving efficiency in the use of energy resources. For that, authors present GeoWorldSim, an agent-based simulation platform that integrates the development of a human activity model as well as the communication middleware known as FIWARE in order to test the best communication architectures available for the implementation of demand-response strategies. The value of the contribution is validated through a simulation scenario.

We gratefully acknowledge the reviewers as well as the programme committee members of 13th IEEE International Conference on Ubiquitous Intelligence and Computing (UIC 2016) for their help in selecting the papers for this special issue. We want to also thank the authors for the effort spent in improving and extending the original proposals. Finally, we would like to thank the referees of this special issue for the high quality of their reviews and also to the editors of the journal for their helpful support.

\section{Lead Guest Editor}

Diego López-de-Ipiña

Guest Editors

Liming Chen

Nathalie Mitton

Gang Pan

\section{References}

1. Triboan D, Chen L, Chen F, Wang Z (2017) Semantic segmentation of real-time sensor data stream for complex activity recognition. Pers Ubiquit Comput. doi:10.1007/s00779-0171005-5

2. Shi D, Wang R, Wu Y, Mo X, Wei J (2017) A novel orientation and location-independent activity recognition method. Pers Ubiquit Comput. doi:10.1007/s00779-017-1007-3

3. Haroun A, Mostefaoui A, Dessables F (2017) Data fusion in automotive applications-efficient big data stream computing approach. Pers Ubiquit Comput. doi:10.1007/s00779-017-1008-2

4. Kabác M, Consel C, Volanschi N (2017) Designing parallel data processing for enabling large-scale sensor applications. Pers Ubiquit Comput. doi:10.1007/s00779-017-1009-1

5. Bermudez-Edo M, Elsaleh T, Barnaghi P, Taylor K (2017) IoTLite: a lightweight semantic model for the internet of things and its use with dynamic semantics. Pers Ubiquit Comput. doi:10.1007/ s00779-017-1010-8

6. Chen Y, Guo M, Shen J, Cao J (2017) GraphLoc: a graph-based method for indoor subarea localization with zero-configuration. Pers Ubiquit Comput. doi:10.1007/s00779-017-1011-7

7. Lopez-Novoa U, Aguilera U, Emaldi M, López-de-Ipiña D, Pérezde-Albeniz I, Valerdi D, Iturricha Arza E (2017) Overcrowding detection in indoor events using scalable technologies. Pers Ubiquit Comput. doi:10.1007/s00779-017-1012-6

8. Ioannidis D, Vidaurre-Arbizu M, Martin-Gomez C, Krinidis S, Moschos I, Zuazua-Ros A, Tzovaras D, Likothanassis S (2017) Comparison of detailed occupancy profile generative methods to published standard diversity profiles. Pers Ubiquit Comput. doi:10. 1007/s00779-017-1013-5

9. Kamara-Esteban O, Pijoan A, Alonso-Vicario A, Borges CE (2017) On-demand energy monitoring and response architecture in a Ubiquit world. Pers Ubiquit Comput. doi:10.1007/s00779-0171014-4 